\title{
Dendritic cells loaded with HeLa-derived exosomes simulate an antitumor immune response
}

\author{
GUOPING REN, YANHONG WANG, SHEXIA YUAN and BAOLIAN WANG \\ Department of Obstetrics, Xinxiang Central Hospital, Xinxiang, Henan 453000, P.R. China
}

Received September 12, 2016; Accepted January 12, 2018

DOI: $10.3892 / \mathrm{ol} .2018 .8126$

\begin{abstract}
The aim of the present study was to investigate the effect of loading dendritic cells (DCs) with HeLa-derived exosomes on cytotoxic T-lymphocyte (CTL) responses, and the cytotoxic effects of CTL responses on the HeLa cell line. Ultrafiltration centrifugation combined with sucrose density gradient ultracentrifugation was applied to isolate exosomes (HeLa-exo) from the supernatant of HeLa cells. Morphological features of HeLa-exo were identified by transmission electron microscopy (TEM), and the expression of cluster of differentiation (CD)63 was detected by western blotting. Next, monocytes were isolated from peripheral blood and cultured with the removal of adherent cells to induce DC proliferation. DCs were then phenotypically characterized by flow cytometry. Finally, MTT assays were performed to analyze the effects of DCs loaded with HeLa-exo on T cell proliferation and cytotoxicity assays to evaluate the effect of CTL responses on HeLa cells. TEM revealed that HeLa-exo exhibit typical cup-shaped morphology with a diameter range of 30-100 nm. It was also identified that the CD63 surface antigen is expressed on HeLa-exo. Furthermore, monocyte-derived DCs were able to express CD1a, suggesting that DC induction was a success. DCs exhibited hair-like protrusions and other typical dendritic cell morphology. Furthermore, DCs loaded with HeLa-exo could enhance CTL proliferation and the cytotoxic activity of CTLs compared with DCs without HeLa-exo $(\mathrm{P}<0.05)$. In conclusion, DCs loaded with HeLa-exo may promote $\mathrm{T}$ cell proliferation and induce CTL responses to inhibit the growth of cervical cancer cells in vitro.
\end{abstract}

\section{Introduction}

Exosomes are vesicles of 30-100 $\mathrm{nm}$ in diameter, which are secreted from eukaryotic cells. Exosomes contain a variety of cell membrane molecules and associated proteins, which

Correspondence to: Professor Baolian Wang, Department of Obstetrics, Xinxiang Central Hospital, 56 Jinsui Road, Xinxiang, Henan 453000, P.R. China

E-mail: baolianwangcn@163.com

Key words: exosomes, HeLa cell lysates, dendritic cells, T cells occupy a lipid bilayer structure, and they are transferred into the extracellular space through fusion of membrane intracellular multivesicular bodies (MVBs) with cell membrane. Many types of cells can release exosomes in this mode of exocytosis $(1,2)$. Tumor-derived exosomes (Texos), released from tumor cells into the extracellular environment, are enriched with tumor-associated antigens, major histocompatibility complex (MHC) molecules, tetraspanin [cluster of differentiation (CD) 9 and CD63] and costimulatory molecules (3). They induce antitumor immune responses of cytotoxic T lymphocytes (CTLs) (4). However, the low efficiency of this process renders it difficult to create an effective cancer treatment exploiting this process. Therefore, it would be beneficial to enhance Texo-induced anti-tumor effects and develop an efficient Texo-based tumor vaccination.

Tumor cells exhibit tumor surface antigens, which cause specific antitumor immunity when immunological cells identify antigens. Dendritic cells (DCs) are the most potent antigen presenting cells (APC), and their primary function is to process and present antigens to stimulate naive $\mathrm{T}$ cell proliferation, and activation (5). This is critical for the execution, control and maintenance of immune responses, including tumor-specific immune responses (4). A previous study demonstrated that Texos target DCs in vitro, and the Texo-DCs enhanced antitumor immunity (6). In recent years, the incidence of cervical cancer in China has been identified as the second highest in gynecological malignant solid tumors, and the age of female patients presenting with cervical cancer has decreased (7). Radiotherapy and chemotherapy are associated with numerous side effects; therefore, novel therapeutic approaches are required for the treatment of cervical cancer.

In the present study, HeLa-exo was isolated from HeLa cells, and co-cultured with DCs to achieve HeLa-exo loading of DCs in vitro. Finally, the present study investigated the effect of HeLa-exo-loaded DC T lymphocyte proliferation and CTL responses for inhibition of the growth of cervical cancer cells. The present study aimed to provide experimental evidence for cervical cancer immunotherapy.

\section{Materials and methods}

Isolation and purification of HeLa-exo. HeLa cells (American Type Culture Collection, Manassas, VA, USA) were cultured in RPMI-1640 medium (Hyclone; GE Healthcare, Chicago, IL, USA) supplemented with $10 \%$ heat-inactivated fetal 
bovine serum (FBS; Si Ji Qing, Hangzhou, China) and incubated at $37^{\circ} \mathrm{C}$ in $5 \% \mathrm{CO}_{2}$ for $2 \mathrm{~h}$. A total of $200 \mathrm{ml}$ cell culture supernatant was collected while cells were in the logarithmic growth phase, and centrifuged at $300 \mathrm{x} \mathrm{g}$ at $4^{\circ} \mathrm{C}$ for $10 \mathrm{~min}$ to remove cells. The supernatant was then centrifuged at $2,000 \mathrm{x} \mathrm{g}$ at $4^{\circ} \mathrm{C}$ for $30 \mathrm{~min}$ to remove dead cells, and again at $10,000 \mathrm{x} \mathrm{g}$ at $4^{\circ} \mathrm{C}$ for $30 \mathrm{~min}$ to remove cell debris. The supernatant was collected and transferred into $100 \mathrm{kD}$ Amicon Ultra-15 ultracentrifuge tubes (Merck KGaA, Darmstadt, Germany) and centrifuged at $1,500 \mathrm{x} \mathrm{g}$ at $4^{\circ} \mathrm{C}$ for $30 \mathrm{~min}$ to the volume of concentrated solution $<5 \mathrm{ml}$. The concentrated solution, $30 \mathrm{~g} / 1$ sucrose/water-cushion and $0.01 \mathrm{~mol} / 1 \mathrm{PBS}$ were added sequentially to Beckman ultracentrifuge tubes (Beckman Coulter, Inc., Brea, CA, USA) at a ratio of 3:3:4, and centrifuged at $100,000 \mathrm{x} \mathrm{g}$ and $4^{\circ} \mathrm{C}$ for $60 \mathrm{~min}$. Next, the HeLa-exo loaded sucrose was collected, diluted x 50 with PBS, and centrifuged at $100,000 \mathrm{x} \mathrm{g}$ at $4^{\circ} \mathrm{C}$ for $60 \mathrm{~min}$ in ultracentrifuge tubes to form a HeLa-exo pellet. The pellet was collected and resuspended in PBS. The exosome protein concentration was determined using a BCA protein assay kit (Wanleibio, Co., Ltd., Shanghai, China), according to the manufacturer's protocol. Finally, the HeLa-exo solution was filtered using a $0.22 \mu \mathrm{m}$-diameter filter membrane (Beckman Coulter, Inc.) and stored at $-80^{\circ} \mathrm{C}$ until use.

Observation of morphological features of HeLa-exo by TEM. Samples of HeLa-exo protein were fixed in $2.5 \%$ glutaraldehyde and rinsed with PBS. The samples were then mounted with $1 \%$ osmium tetroxide, and washed twice with PBS. The samples were then dehydrated using a gradient of acetone and ethanol (30\% ethanol, $50 \%$ ethanol, $70 \%$ ethanol, $80 \%$ acetone, $90 \%$ acetone and $100 \%$ acetone), impregnated with epoxy resin, embedded and polymerized with paraffin. Finally, the samples were sliced into $70 \mathrm{~nm}$-ultrathin sections for $3 \%$ uranyl acetate-lead citrate staining at $37^{\circ} \mathrm{C}$ for $30 \mathrm{~min}$, and observed with a JEM-1200EX transmission electron microscope (magnification, x20,000) (JEOL, Ltd., Tokyo, Japan).

Western blot analysis. The concentration of HeLa cells was diluted in RPMI-1640 medium (Hyclone; GE Healthcare, Chicago, IL, USA) and adjusted to $1 \times 10^{8}$ cells $/ \mathrm{ml}$, and after 4 rapid freeze-thaw cycles at $-20^{\circ} \mathrm{C}$, the cells and media were centrifuged at $1,000 \mathrm{x} \mathrm{g}$ at $4^{\circ} \mathrm{C}$ for $30 \mathrm{~min}$, providing a supernatant containing the cell lysate. A BCA protein assay was used to calculate the protein concentration of the supernatant (Wanleibio, Co., Ltd.), according to the manufacturer's protocol. A total of $40 \mu \mathrm{g}$ HeLa-exo or untreated HeLa cell lysate were subjected to $12 \%$ SDS-PAGE, and the separated proteins were transferred onto cellulose acetate membranes. Next, the membranes was blocked in 5\% skim milk at room temperature for $2 \mathrm{~h}$, and incubated with anti-CD63 (cat. no. PB0236; dilution, 1:500) and anti- $\beta$-actin (cat. no. BM0627; dilution, 1:500) (both Boster Biological Technology, Pleasanton, CA, USA) at $4^{\circ} \mathrm{C}$ overnight. The membranes were then washed thrice with Tris-PBS, prior to incubation with a horseradish peroxidase-labeled goat anti-rabbit secondary antibody (cat. no. BA1050; dilution, 1:5,000; Boster Biological Technology) at $37^{\circ} \mathrm{C}$ for $30 \mathrm{~min}$. The membranes were washed thrice with Tris-PBS, and ECL luminescent liquid (cat. no. 34577; Thermo Fisher Scientific, Inc., Waltham, MA, USA) was used for exposure and development, according to the manufacturer's protocol.

Induction of DCs from monocytes. All the samples were obtained from 18 patients with cervical cancer, who were admitted to the Department of Obstetrics, Xinxiang Central Hospital (mean age, 53.8 \pm 3.6 years) between January 2015 and June 2015 who provided informed consent, and the following experiments were approved according to Xinxiang Central Hospital (Xinxiang, China) guidelines. A total of $30 \mathrm{ml}$ each fresh human peripheral blood sample from 18 patients was divided evenly and placed into separate $50 \mathrm{ml}$-centrifuge tubes. PBS was added to reach a volume of $35 \mathrm{ml}$, then $15 \mathrm{ml}$ Ficoll separating medium (Dingguo, Beijing, China) was added to the bottom of the tubes. The solutions were centrifuged at $300 \mathrm{x} \mathrm{g}$ at room temperature for $20 \mathrm{~min}$. The lymphocyte-containing second layer was collected and placed into a centrifuge tube. Following the addition of $5 \mathrm{ml}$ PBS, it was further centrifuged at $300 \mathrm{x} \mathrm{g}$ at room temperature for $25 \mathrm{~min}$, and then the supernatant was discarded. The remaining cells were rinsed in PBS and counted on a hemocytometer. D14+ cells were isolated using a CD14 cell magnetic activated cell sorting system (Miltenyi Biotec $\mathrm{GmbH}$, Bergisch Gladbach, Germany), according to the manufacturer's protocol. CD14-cells were stored at $-80^{\circ} \mathrm{C}$. CD14+ cells were placed in complete RPMI-1640 medium (Hyclone; GE Healthcare, Chicago, IL, USA, Logan, Utah, USA) supplemented with 10\% heat-inactivated FBS (Si Ji Qing, Hangzhou, China) and counted. Recombinant human granulocyte-macrophage colony stimulating factor, recombinant human interleukin 4 and tumor necrosis factor $\alpha$ were all added at 1,000 IU/ml (all from PeproTech, Inc., Rocky Hill, NJ, USA). Cells were then seeded in 12-well cell culture plates at $1 \times 10^{6}$ cells/well, and the same concentrations of growth factors were added after 3 days. After being cultured for 7 days, $1 \times 10^{5}$ cells were collected for analysis of CD1a expression by flow cytometry. Finally, 2 wells were selected for loading of HeLa-exo $(10 \mu \mathrm{g})$. The cells were cultured with HeLa-exo for $48 \mathrm{~h}$, prior to harvesting of HeLa-exo-DCs.

$T$ cell proliferation assay. Frozen CD14- cells were recovered and resuspended in RPMI-1640 medium prior to being filtered through a nylon mesh to separate the T cells. After $1 \mathrm{~h}$ in conventional culture, DCs and T lymphocytes (Ts) were cultured at a ratio of 1:3. This assay included 3 groups: A, HeLa-exo-DC+T; B, DC+T; and C, T (negative control). The cells were plated at $100 \mu \mathrm{l}\left(1 \times 10^{5}\right.$ cells $) /$ well in a $96-w e l l$ culture plate, with six replicates for each group. The plate was kept at $37^{\circ} \mathrm{C}$ in $5 \% \mathrm{CO}_{2}$. After 3 days, $20 \mu \mathrm{l}$ MTT was added to each well and the plate was incubated for another $4 \mathrm{~h}$. A total of $150 \mu$ l DMSO (Sigma-Aldrich; Merck KGaA) was then added into each well and shaken gently to dissolve the formazan crystals. Absorbance (A) values were measured by a spectrophotometer at $490 \mathrm{~nm}$.

Cytotoxicity assay. This assay included four groups: Experimental group [HeLa-exo-DCs+T (1:3)], effector cell control group (T), target cell control group (HeLa-exo+T), DC negative control group (DCs+T), with T lymphocytes regarded as effector cells. Next, cells in the experimental group were 


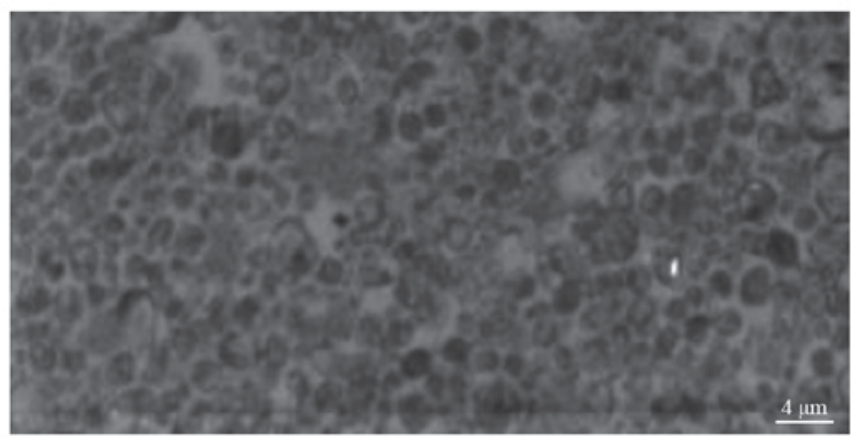

Figure 1. Electron micrograph of exosomes secreted from HeLa cells (x20,000 magnification). HeLa-derived exosomes were circular or elliptical with relatively uniform size.

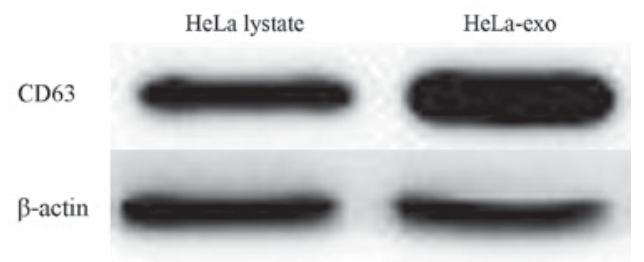

Figure 2. Western blot analysis of CD63 protein expression levels by HeLa cell-derived exosomes and untreated HeLa cell lysate. Both HeLa-exo and HeLa cell lysates expressed CD63 molecules, and the CD63 expression on tumor-derived exosome was increased compared with that on tumor cell lysates. CD, cluster of differentiation.

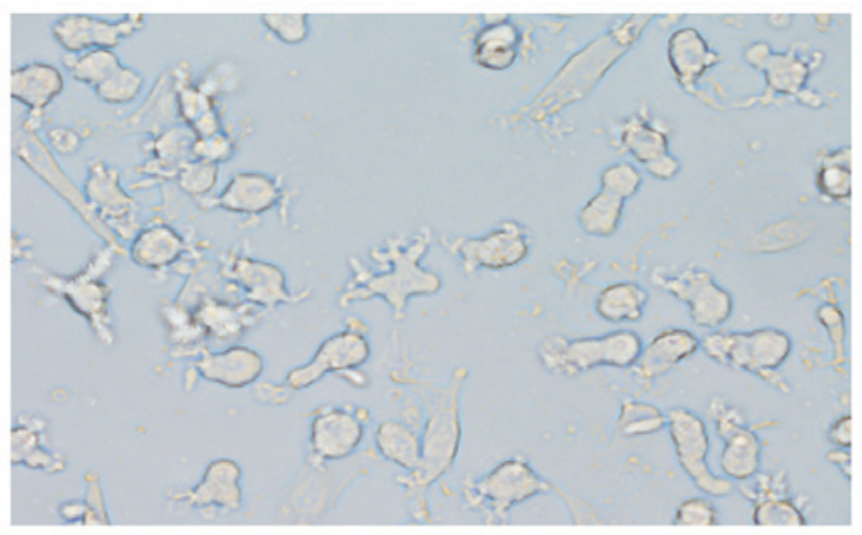

Figure 3. Photomicrograph demonstrating the morphological features of cluster of differentiation $14+$ cells at 7 days of culture (x400 magnification). After 7 days of culture, adherent CD14 + cells aggregates were visible under a light microscope.

plated at $100 \mu \mathrm{l}\left(1 \times 10^{5}\right.$ cells $) /$ well in a 96-well culture plate containing HeLa cells. Experiments were performed with ratios of effector:target cells of 12.5:1, 25:1 and 50:1, and each group had three replicates. The plate was incubated with interleukin-2 (400 U/ml) (Boster Biological Technology) at $37^{\circ} \mathrm{C}$ in $5 \% \mathrm{CO}_{2}$. After $48 \mathrm{~h}, 20 \mu \mathrm{l}$ MTT was added to each well, and the plate was incubated for another $4 \mathrm{~h}$. A total of $150 \mu \mathrm{l}$ DMSO (Sigma-Aldrich; Merck KGaA) was then added to each well, and the plate was gently agitated at $37^{\circ} \mathrm{C}$ for $10 \mathrm{~min}$. Absorbance (A) values were measured by a spectrophotometer at $570 \mathrm{~nm}$, and used to calculate the percentages of cytotoxicity according to the following formula: [A value

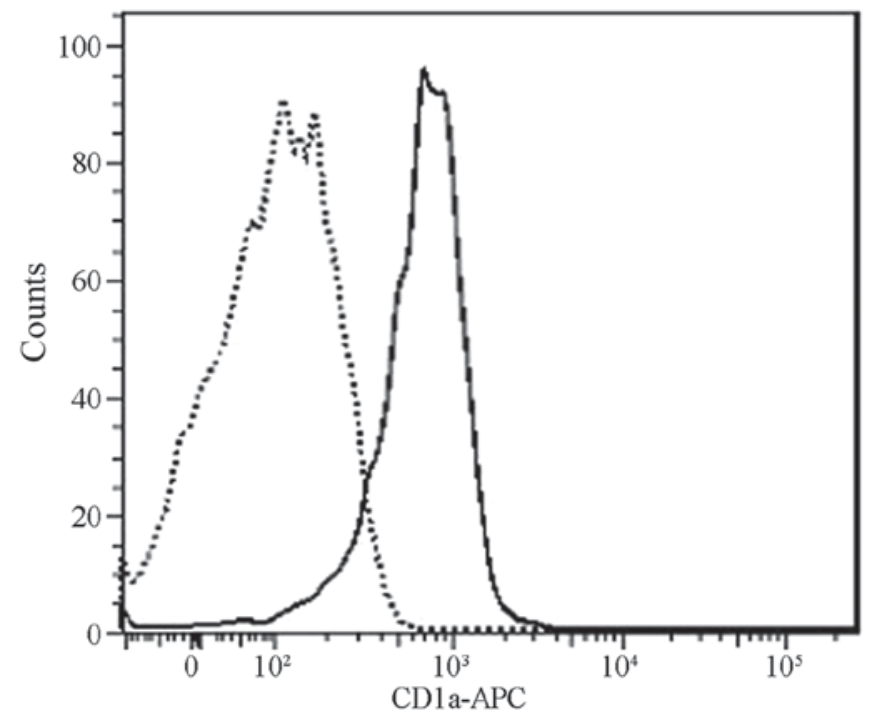

Figure 4. Flow cytometry analysis of cluster of differentiation 1a (CD1a) surface expression on dendritic cells. The dotted line represents an isotype control expression and the solid line represents CD1a expression.

of HeLa-exo+T group-(A value of HeLa-exo-DCs+T group-A value of $\mathrm{T}$ group)/A value of HeLa-exo+T group] x100.

Statistical analysis. All statistical analyses were performed using SPSS 17 software (SPSS, Inc., Chicago, IL, USA). Measurement data is expressed as mean \pm standard deviation. Comparisons between 2 groups were performed using Student's t-test, while comparisons among $\geq 3$ groups were analyzed using one-way analysis of variance followed by the post hoc test with Student-Newman-Keuls test. $\mathrm{P}<0.05$ was considered to indicate a statistically significant difference. A $\chi^{2}$ test was also performed on cytotoxicity data, and the significance threshold of pairwise comparison was adjusted to $\mathrm{P}<0.01$ with Bonferroni correction method.

\section{Results}

Identification of HeLa-exo. As demonstrated in Fig. 1, HeLa-derived exosomes exhibited a circular or elliptical shape, were relatively uniform in size and had a diameter of $30-100(57.3 \pm 4.2) \mathrm{nm}$. HeLa-exo were surrounded by a lipid membrane and had an inner low electron density membrane. Both HeLa-exo and HeLa cell lysates expressed CD63; however, CD63 expression of the tumor-derived exosomes was markedly higher compared with that of tumor cell lysates (Fig. 2).

DC phenotyping. After 7 days of culture, adherent CD14+ cell aggregates were visible under a light microscope, presenting typical dendritic processes of different sizes, and some of the cells were forming colonies (Fig. 3). As demonstrated in Fig. 4, flow cytometry analysis detected CD1a marker expression on the surface of the cells, indicating that the DC induction was successful.

DCs loaded with HeLa-exo stimulate T cell proliferation. As presented in Fig. 5 , the number of T cells in the HeLa-exo-DC+T 
Table I. Percentage inhibition of HeLa cell growth following induction of CTL activity by Hela-exo loaded DCs.

\begin{tabular}{lccccc}
\hline & \multicolumn{4}{c}{ Percentage inhibition } \\
\cline { 2 - 5 } Effector:target ratio & A: T & B: Hela-exo+T & C: DCS+T & D: Hela-exo-DCs+T & $\chi^{2}($ P-value $)$ \\
\hline $12.5: 1$ & 7.3 & $3.1^{\mathrm{a}}$ & $27.2^{\mathrm{a}, \mathrm{b}}$ & $38.1^{\mathrm{a}-\mathrm{c}}$ & $53.556(<0.001)$ \\
$25: 1$ & 16.3 & $12.5^{\mathrm{a}}$ & $38.3^{\mathrm{a}, \mathrm{b}}$ & $41.6^{\mathrm{a}, \mathrm{b}}$ & $33.626(<0.001)$ \\
$50: 1$ & 22.2 & 23.8 & $39.3^{\mathrm{a}, \mathrm{b}}$ & $58.5^{\mathrm{a}-\mathrm{c}}$ & $37.193(<0.001)$ \\
\hline
\end{tabular}

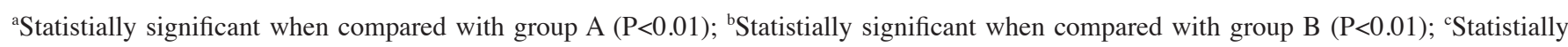
significant when compared with group $\mathrm{C}(\mathrm{P}<0.01)$. DC, dendritic cell; T, T cells; HeLa-exo, HeLa-derived exosomes.

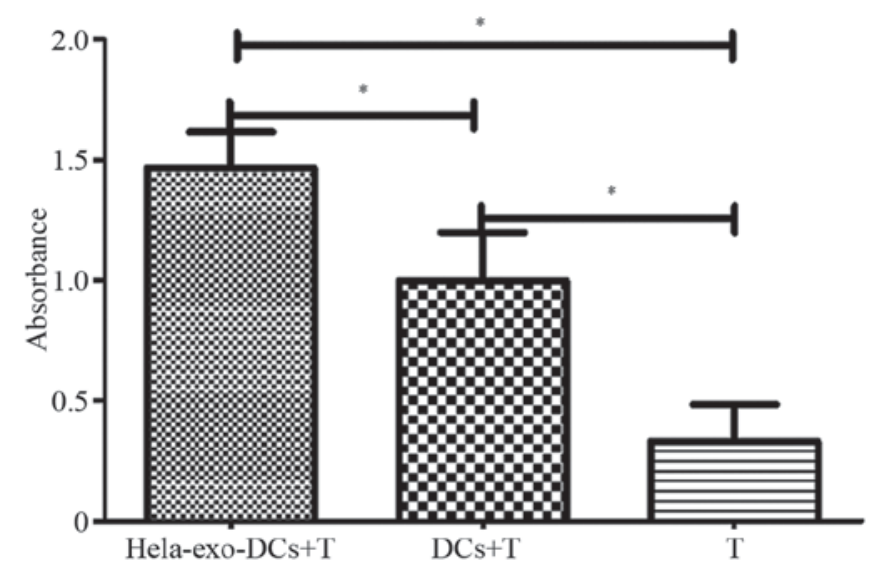

Figure 5. An MTT assay was performed to analyze the effect of dendritic cells loaded with HeLa-exo on T cell proliferation. "P $<0.05$. Results demonstrated that loading DC with HeLa-exo significantly enhanced the ability to stimulate $\mathrm{T}$ cell activation compared with DCs without HeLa-exo loading.

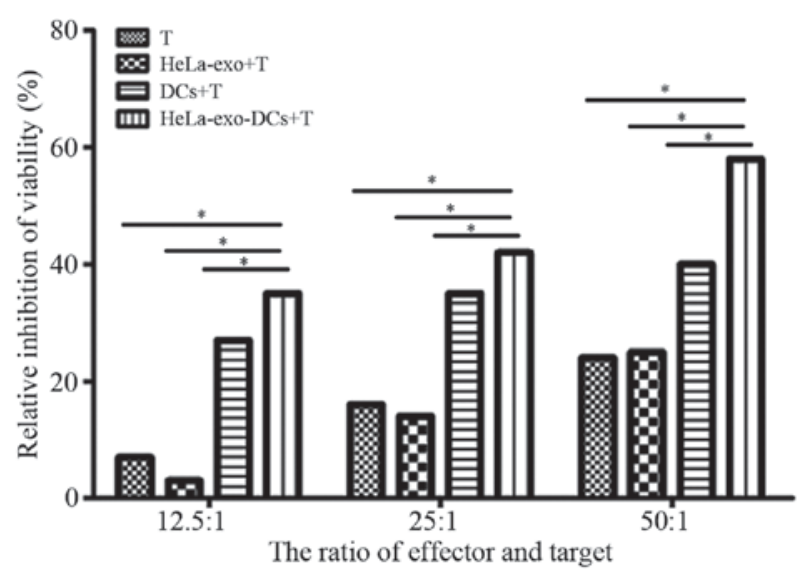

Figure 6. Induction of specific CTL activity induced by HeLa-exo loaded DCs was measured using an MTT assay. ${ }^{*} \mathrm{P}<0.01$. Results demonstrated that anti-Hela activity induced by DC loaded with Hela-exo in Hela-exo-DCs $+\mathrm{T}$ group was significantly increased compared with that of the other three groups $(\mathrm{P}<0.01)$.

and $\mathrm{DC}+\mathrm{T}$ groups increased significantly compared with the $\mathrm{T}$ group $(\mathrm{P}<0.05)$. Furthermore, the number of $\mathrm{T}$ cells identified in the HeLa-exo-DC+T group was significantly increased compared with the $\mathrm{DC}+\mathrm{T}$ group $(\mathrm{P}<0.05)$. This indicates that loading DC with HeLa-exo significantly enhanced the ability to stimulate $\mathrm{T}$ cell activation compared with DCs without HeLa-exo loading (Fig. 5).

HeLa-exo-DCs induce CTL specific cytotoxicity. As demonstrated in Table I, with an effector:target ratio of 12.5:1, 25:1 or 50:1, the anti-HeLa activity induced by DCs loaded with HeLa-exo in HeLa-exo-DCs+T group was significantly increased compared with all other groups $(\mathrm{P}<0.01$; Table I; Fig. 6).

\section{Discussion}

Cervical cancer is one of the most common types of malignant tumor identified in women (8). Although conventional surgical treatment may effectively remove visible tumor masses, surgery does not ensure the removal of all small lesions or metastases. Radiotherapy and chemotherapy have limited efficacy as adjuvant therapies for patients who are resistant to chemotherapy drugs or who cannot tolerate their side effects. Immunotherapy remains as a treatment option. DC tumor vaccines may efficiently deliver specific antigens for naïve $\mathrm{T}$ cell activation for antigen-specific CTL responses, in order to induce lasting tumor-specific immune responses in patients with cervical cancer (9).

Tumor-derived exosomes have been a popular research topic in recent years due to their potential to be used in anti-tumor vaccines $(3,4)$. An exosome is a microcapsule membranous structure secreted from living cells and its functions vary depending on the cell source (10-12). Previous studies have primarily focused on exosomes derived from tumor cells and DCs. Tumor-derived exosomes, containing MHC-I molecules, tetraspanin (CD9 and CD63) and tumor-associated antigens, activate $\mathrm{T}$ lymphocyte proliferation and CTL responses, but only at low levels $(13,14)$. However, following DC loading, such exosomes are able to activate $\mathrm{T}$ lymphocyte responses, which serve anti-tumor immune effects $(15,16)$.

In the present study, ultrafiltration centrifugation and sucrose density gradient ultracentrifugation were applied to isolate HeLa-exo from the supernatant of HeLa cells. Morphological investigation by TEM revealed that HeLa-exo exhibited a circular or elliptical shape and a relatively uniform size, with a diameter of 30-100-nm. The cells were surrounded by a lipid outer-membrane and a low electron density inner-membrane. Furthermore, expression of CD63 molecules was visible on the surface of DCs, which was consistent with the results of previous literature $(17,18)$. Subsequent to cytokine 
DC induction from peripheral blood mononuclear cells derived from patients with cervical cancer, the DCs were loaded with HeLa-exo in vitro. The present study investigated the effects of DCs loaded with HeLa-exo on $\mathrm{T}$ cell activation and proliferation, and the cytotoxic effects of activated $\mathrm{T}$ cells on cervical cancer cells. The results demonstrated that HeLa-exo alone was not able to stimulate $\mathrm{T}$ cell proliferation, but DCs loaded with HeLa-exo and DCs without HeLa-exo were able to stimulate $\mathrm{T}$ cell proliferation. DCs loaded with HeLa-exo had an enhanced ability to stimulate $\mathrm{T}$ cell activation compared with DCs without HeLa-exo. Cytotoxicity assays revealed that DCs loaded with HeLa-exo had a strong cytotoxic ability compared with the T group and DCs without HeLa-exo had no significant anti-tumor effect compared with the $\mathrm{T}$ group. These results suggest that HeLa-exo-DC induces T lymphocyte activation, and that activated CTLs exhibit an anti-tumor effect, which inhibits the growth of HeLa cells.

HeLa-exo alone are insufficient to induce CTL responses, because T cell activation required DCs, as well as MHC-1, and tumor antigens, to provide costimulatory signals. Thus, only when loaded into DCs could HeLa-exo efficiently induce the CTL anti-tumor effect.

In conclusion, the present study successfully isolated exosomes from HeLa cells, and confirmed that DCs loaded with HeLa-derived exosomes induce CTL activity, enhancing the anti-tumor immune response. This provides basis for research into HeLa-exo use for cancer immunotherapy.

\section{References}

1. Lu M, Huang B, Hanash SM, Onuchic JN and Ben-Jacob E: Modeling putative therapeutic implications of exosome exchange between tumor and immune cells. Proc Natl Acad Sci USA 111: E4165-E4174, 2014.

2. Hannafon BN and Ding WQ: Intercellular communication by exosome-derived microRNAs in cancer. Int J Mol Sci 14: 14240-14269, 2013.

3. Duvallet E, Boulpicante M, Yamazaki T, Daskalogianni C, Prado Martins R, Baconnais S, Manoury B, Fahraeus R and Apcher S: Exosome-driven transfer of tumor-associated Pioneer Translation Products (TA-PTPs) for the MHC class I cross-presentation pathway. Oncoimmunology 5: e1198865, 2016.
4. Tauro BJ, Greening DW, Mathias RA, Mathivanan S, Ji H and Simpson RJ: Two distinct populations of exosomes are released from LIM1863 colon carcinoma cell-derived organoids. Mol Cell Proteomics 12: 587-598, 2013.

5. Shang N, Figini M, Shangguan J, Wang B, Sun C, Pan L, Ma Q and Zhang Z: Dendritic cells based immunotherapy. Am J Cancer Res 7: 2091-2102, 2017.

6. Marleau AM, Chen CS, Joyce JA and Tullis RH: Exosome removal as a therapeutic adjuvant in cancer. J Transl Med 10: 134, 2012.

7. Li H, Duan D, Xu J, Gong Q, Wang Y, Ji W, DU L, Han L and $\mathrm{Xu}$ G: The incidence and mortality of cervical cancer in ningbo during 2006-2014, China. Iran J Public Health 46: 1324-1331, 2017.

8. Jiang $\mathrm{X}$, Tang $\mathrm{H}$ and Chen T: Epidemiology of gynecologic cancers in China. J Gynecol Oncol 29: e7, 2018.

9. Santos PM and Butterfield LH: Dendritic cell-based cancer vaccines. J Immunol 200: 443-449, 2018.

10. Wahlgren J, Karlson Tde L, Glader P, Telemo E and Valadi H: Activated human $\mathrm{T}$ cells secrete exosomes that participate in IL-2 mediated immune response signaling. PLoS One 7: e49723, 2012.

11. King HW, Michael MZ and Gleadle JM: Hypoxic enhancement of exosome release by breast cancer cells. BMC Cancer 12: 421, 2012.

12. Wei Y, Li M, Cui S, Wang D, Zhang CY, Zen K and Li L: Shikonin inhibits the proliferation of human breast cancer cells by reducing tumor-derived exosomes. Molecules 21: pii: E777, 2016.

13. Hayoun D, Kapp T, Edri-Brami M, Ventura T, Cohen M, Avidan A and Lichtenstein RG: HSP60 is transported through the secretory pathway of 3-MCA-induced fibrosarcoma tumour cells and undergoes N-glycosylation. FEBS J 279: 2083-2095, 2012.

14. Hartman ZC, Wei J, Glass OK, Guo H, Lei G, Yang XY, Osada T, Hobeika A, Delcayre A, Le Pecq JB, et al: Increasing vaccine potency through exosome antigen targeting. Vaccine 29: 9361-9367, 2011

15. Marton A, Vizler C, Kusz E, Temesfoi V, Szathmary Z, Nagy K, Szegletes Z, Varo G, Siklos L, Katona RL, et al: Melanoma cell-derived exosomes alter macrophage and dendritic cell functions in vitro. Immunol Lett 148: 34-38, 2012.

16. Bu N, Wu H, Sun B, Zhang G, Zhan S, Zhang R and Zhou L: Exosome-loaded dendritic cells elicit tumor-specific CD8+ cytotoxic T cells in patients with glioma. J Neurooncol 104: 659-667, 2011.

17. Sung BH, Ketova T, Hoshino D, Zijlstra A and Weaver AM: Directional cell movement through tissues is controlled by exosome secretion. Nat Commun 6: 7164, 2015.

18. del Cacho E, Gallego M, Lillehoj HS, Quilez J, Lillehoj EP and Sánchez-Acedo C: Tetraspanin-3 regulates protective immunity against Eimeria tenella infection following immunization with dendritic cell-derived exosomes. Vaccine 31: 4668-4674, 2013. 